\title{
DISKY ELLIPTICALS IN THE HUBBLE SEQUENCE
}

\author{
CECILIA SCORZA \\ Landessternwarte \\ Königstuhl, D-69117 Heidelberg, Germany \\ AND \\ RALF BENDER \\ Universitätssternwarte \\ Scheinerstr. 1, D-81679 München, Germany
}

\section{Introduction}

About one third of the elliptical galaxies show disky isophotes (Bender et al. 1989). These are low luminosity objects, are rotationally flattened (Carter 1987, Bender 1988b, Nieto 1988), are much weaker radio emitters than ellipticals in average and show no strong X-ray emission in excess of their discrete sources contribution. They have pointed isophotes which are thought to reflect the presence of embedded stellar disks. A large number of these disks could be hidden due to low inclinations (Rix and White 1990). Because of these properties, which makes them strongly resemble S0 galaxies, it has been suggested that disky ellipticals form together with S0s and spirals a continuous transition in D/B ratio in the Hubble sequence (Bender 1988b, Nieto 1988). However, until the present, this hypothesis had not been investigated throughout.

The aim of the present study is to place the E-S0 link on a more quantitative base. As a first step, we investigate whether the observed photometric and kinematic properties of these galaxies can be understood within the frame of work of bulge+disk models. Then we quantify the link between disky Es and S0 galaxies in terms of their D/B ratios. An important question to be addressed in this context is to what extend the observed kinematics of disky Es are influenced by the embedded disks. Once the kinematic contribution of the disks is separated, are the bulges of these objects rotationally flattened or are they anisotropic? In doing so, we hope to derive constraints that help to answer the following question: Is the dichotomy in 
in the elliptical family indicating two different origins for disky and boxy ellipticals? or in other words, did all ellipticals form from mergers?

\section{The Internal Structure of Disky Elliptical Galaxies}

In order to investigate the internal structure of the galaxies and see whether their properties can be understood within disk+bulge models, a photometric and kinematic decomposition was applied to a sample of representative objects (for the description of the methods and results see Scorza and Bender 1990,1995). The photometric decomposition allowed to determine the disk parameters, namely the central surface brightness $S B_{0}$, the scale length $r_{0}$ and inclination $i$. The kinematic decomposition relies on the fact that the velocity line profiles (VLP) of disky ellipticals are asymmetric (Bender 1990, Bender, Saglia and Gerhard 1994). We find that the radii at which the asymmetries are largest corresponds to the radii at which the disks constribute with most of their light (see Fig 5 in Scorza and Bender 1995). In view of this, a double-gaussian fit to the VLPs was carried out under the constraint that the flux ratio of the two Gaussians is similar to the $\mathrm{D} / \mathrm{B}$ derived from the photometry. The results do not change if we allow for a restricted range of intrinsic asymmetries in the VLPs of the bulges. We find that the VLPs of the galaxies can be very nicely modelled and that the kinematic contribution of the disk and bulge components can be, in first approximation, separated.

\section{The continuity of physical properties between E-SO Galaxies}

\subsection{D/B RATIO-SEQUENCE BETWEEN E AND S0 GALAXIES}

Fig 1.a shows a histogram of the D/B ratios determined by Kent (1985) for S0 and spiral galaxies together with the values derived here from the photometric decomposition of disky ellipticals. The dependence of the $\mathrm{D} / \mathrm{B}$ ratios on the Hubble type is clear. To better visualize the distribution of disky ellipticals, a similar histogram is shown in Fig 1.b but covering only the range $D / B<1$. It can be seen that the $\mathrm{D} / \mathrm{B}$ ratios of disky ellipticals are confined to $<0.20$. 
(a)

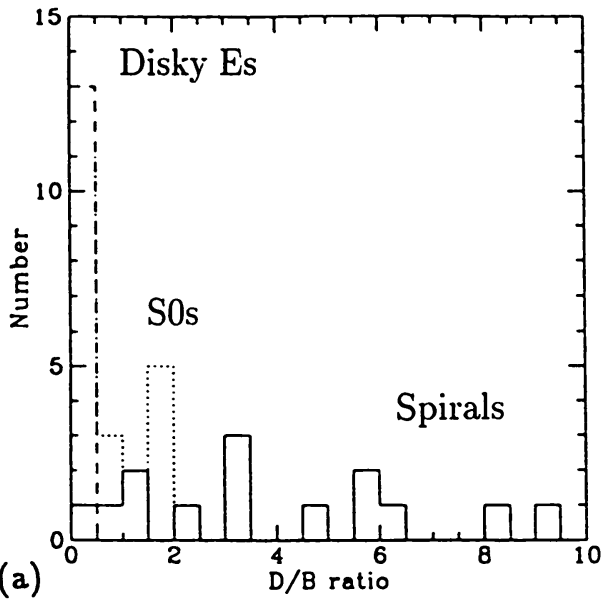

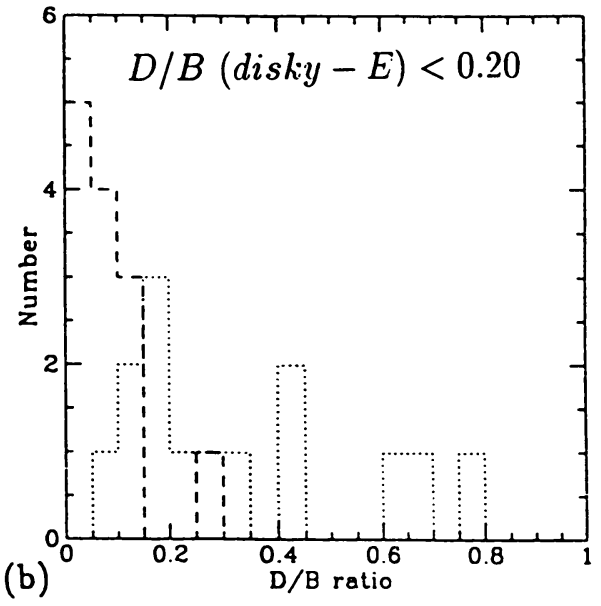

Figure 1. (a) The D/B ratios estimated by Kent (1985) for spiral (solid line) and S0 galaxies (pointed lines) together with the values determined here for disky ellipticals (dashed lines). (b) the same as (a) but confined to D/B ratios $<1$

\subsection{BULGE AND DISK LUMINOSITIES ALONG THE HUBBLE SEQUENCE}

The behaviour of the bulge and disk luminosities along the Hubble sequence was also investigated. Figures 2.a and 2.b show a plot of the absolute magnitudes of the bulges and disks as a function of the $\mathrm{D} / \mathrm{B}$ ratios. It was found that while the bulge luminosities increase from spirals towards S0s and reach a constant value among S0 and disky Es, the disk luminosities decrease along the entire sequence. This suggests that disks in ellipticals could have formed in a similar way as disks in S0s (we refer here to intrinsic formation theories) with the difference that in the disky $\mathrm{E}$ case, only small amounts of gas ( $10 \%$ of the whole mass) led to the formation of the disks.

\section{Conclusions and Implications for Formation Scenarios of Disky Elliptical Galaxies}

The present study indicates that there is a continuous sequence in $\mathrm{D} / \mathrm{B}$ ratio between E-S0 galaxies. It was found that the angular momentum vectors of disks and bulges are aligned in nearly all objects, which excludes random accretion events as the origin of the disks and therefore supports the connection to $\mathrm{S} 0$ galaxies. After disk subtraction, the bulges are still rotating. A correlation was found indicating that more compact bulges are more rotationally flattened than less compact ones (see Fig. 18 in Scorza and Bender 1995). We find that bulges of galaxies showing merger signatures deviate from this correlation and conclude that those objects following the correlation have most likely an undirsturbed history. 

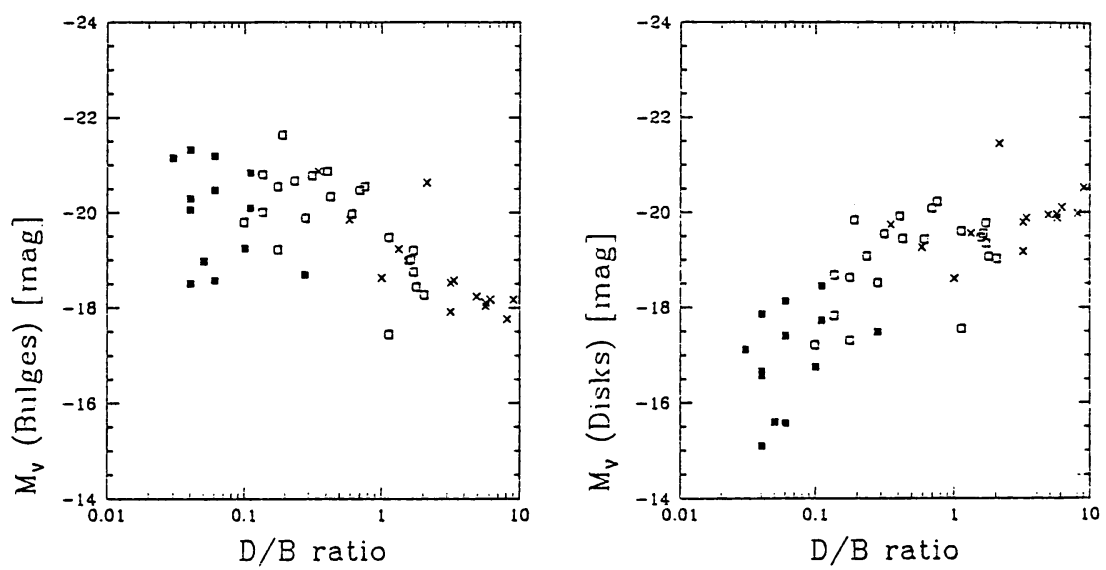

Figure 2. (a) Absolute magnitudes of the bulges and (b) of the disks as a function of the D/B ratios for spirals (crosses), S0 galaxies (open squares) and disky ellipticals (filled squares)

\subsection{DID DISKY ELLIPTICALS FORM FROM MERGERS?}

Collisionless mergers: this scenario cannot explain the high phase-space densities (Carlberg 1987) and unresolved cores of disky ellipticals (Kormendy 1987, Kormendy et al. 1995). Moreover, Steinmetz and Buchner (1995) have recently investigated the kinematic properties of merger remnants in dissipationless simulations. They find that all remnants are anisotropic and most of them boxy, and conclude that disky ellipticals could not have formed in collisionless merger events.

Mergers "con gas": this type of mergers give account for the formation of kinematic sub-components in elliptical galaxies (counter-rotating cores and central disks) as shown by Hernquist and Barnes (1991) and Barnes (1993). The question whether it could also be possible to form the rotationally flattened bulges of disky ellipticals in this way, is still open. Barnes (1993) has shown how small amounts of gas can change the orbit structure of galaxies during mergers; namely the box and $\mathrm{X}$-tube orbits are transformed into Z-tube orbits and the remnant becomes a more oblate structure. A clearer answer will be given by measuring the $V / \sigma$ ratio of the bulges of these remnants and by comparing them with the observed values.

\section{References}

Barnes, J.E., 1993, The Formation of Galaxies, proceedings of the V Canary Islands Winter School of Astrophysics, ed. Munoz-Tunon, C., Cambridge University Press

Bender, R., 1988b, Astr. Ap., 202, L5

Bender, R., Surma, P., Döbereiner, S., Möllenhoff, C., Madejsky, R., 1989, Astr. Ap., 217, 35 
Bender, R., 1990, Astr. Ap., 229, 441.

Bender, R., Saglia, R.P, Gerhard, O.E., 1994, MNRAS, 269, 785

Carlberg, R.G., 1986, Ap. J., 310, 593

Carter, 1987, Ap. J., 312, 514

Hernquist, L., Barnes., J.E., 1991, Letters to Nature 354, 210

Kormendy, J., 1987, in the IAU Symposium 127 Structure and Dynamics of Elliptical Galaxies, eds. T. de Zeeuw

Kormendy, J., et al. 1995, this proceedings

Kent, S., 1985, Ap. J. Suppl. Series, 59, 115

Nieto, J-L., 1988, $2^{\text {da }}$ Reunion de Astronomia Extragalactica, Academia Nacional de Ciencias de Cordoba, Cordoba Argentina, p. 239

Rix, H.-W., White, S., 1990, Ap. J., 362, 52

Scorza, C., Bender, R., 1990, Astr. Ap., 235, 49

Scorza, C., Bender, R., 1995, Astr. Ap., 293, 20

Steinmetz, M., Buchner, S., 1995, in preparation

\section{DISCUSSION}

Rich: How do bulges of ellipticals compare with spiral bulges?

Scorza: From the kinematic point of view, most bulges of spirals, S0s and disky ellipticals are rotationally flatenned. There exists also a smooth trend in absolute luminosities, the bulges of spirals being less luminous than those of S0 and ellipticals (see Fig. 2.a in this contribution).

Zepf: Is there evidence for an environmental dependence in the $\mathrm{D} / \mathrm{B}$ ratio for early-type galaxies?

Scorza: An answer to this question will be provided after an analyses of a larger sample. Segregration studies are on the way in the SternwarteMünchen, under the supervision of Ralf Bender.

Forbes: Is there any evidence for an age difference between the disk and the bulge in an individual elliptical galaxy, say from colors?

Scorza: We haven't look systematically into the colors of the galaxies. There are some evidences for the disks being younger as the bulges in some objects, as indicated by the higher $H_{\beta}$ absorbtion along the major axis (where the disks lie). de Jong et al. (see this conference) have found also a correlation between $H_{\beta}$ absorbtion and diskyness, suggesting the same result.

Huizinga: Do the exponential disks extend all the way to the centre, or do some show central holes?

Scorza: We find both types of behaviour. However most of all objects have disk profiles which extend all the way to the center but deviate sometimes from the exponential law. They seem to follow more an $r^{1 / 4}$ profile. Note however, that seeing makes conclusions concerning the central $S B_{0}$ quite uncertain. 


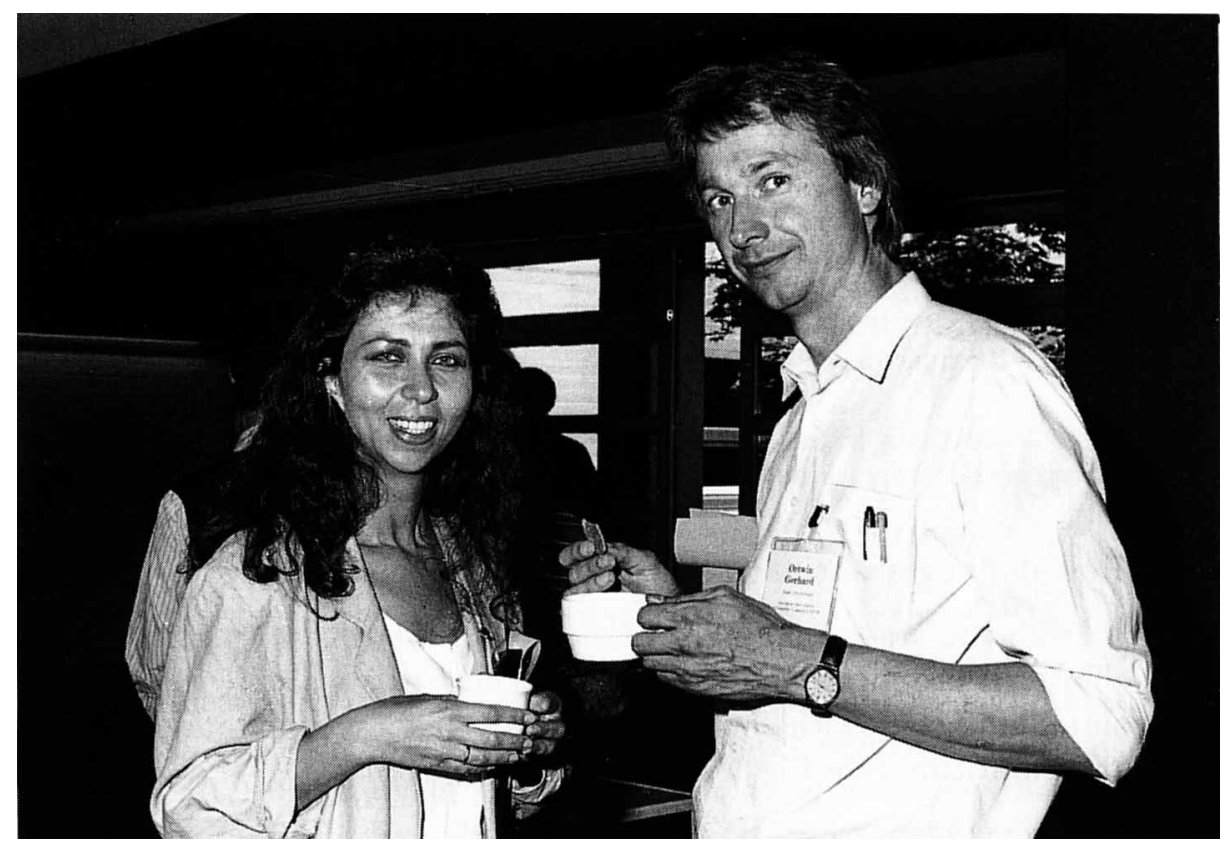

Cecilia Scorza and Ortwin Gerhard 